\title{
Reconnaissance et confiance, une nouvelle étape pour la revue
}

Une dimension symbolique toute particulière s'attache à la livraison de ce trente-deuxième numéro de "Pédagogie Médicale ", le dernier à paraître sous l'égide de son éditeur historique, la société Acalis. Dans le contexte international d'une production scientifique de plus en plus concurrentielle, au sein de laquelle la langue anglaise est survalorisée de façon quasi hégémonique - facteur d'impact oblige -, il fallait être plutôt téméraire pour prendre le risque d'éditer et de soutenir, à l'orée d'un troisième millénaire irréversiblement mondialisé, une revue internationale francophone consacrée à un champ alors encore confidentiel au sein des sciences de la santé, celui de l'éducation et de la pédagogie.

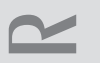

Il faut donc très chaleureusement saluer et remercier Gérard Dongradi, directeur de la publication, mais tout particulièrement sa partenaire entrepreneuriale Mireille Menestrel, secrétaire générale de la revue, d'avoir accepté d'affronter les risques et les vicissitudes d'une aventure stimulante mais incertaine et d'avoir su relever le défi que lui avaient proposé les trois fondateurs du journal, Raymond Colin, JeanFrançois Denef et Bernard Charlin. Leur fidélité sans faille et leur compétence jamais prise en défaut ont été de solides appuis pour toute l'équipe rédactionnelle. Il faut bien sûr associer à ces remerciements tous les membres de leur équipe éditoriale et technique, parmi lesquels, tout spécialement, Cécile Blévat, secrétaire de rédaction, dont la disponibilité et la réactivité ont été si précieuses pour garantir, malgré des "bouclages" effectués parfois à la dernière limite, une parution trimestrielle sans un seul jour de retard durant huit années.

Il faut aussi saluer tous les membres de la cellule infographique de l'Université catholique de Louvain à Bruxelles, et notamment Philippe Gathy, qui ont conçu, mis en cuvre puis entretenu, avec un savoirfaire irréprochable, le site Internet de la revue, élément si important pour la reconnaissance du journal, notamment grâce à la mise en accès libre de tous les articles après une période d'embargo limitée à deux ans.

Grâce à ces partenaires de la première heure, "Pédagogie Médicale " est aujourd'hui une revue pérenne, indépendante et désormais bien identifiée dans le paysage francophone en éducation des sciences de la santé. Pour autant, il nous faut prendre toute la mesure des profondes mutations que vit actuellement l'édition scientifique. Dans un environnement extrêmement compétitif et réactif, les ressources techniques qui la sous-tendent ont été profondément renouvelées. Parallèlement, les modèles économiques qui en permettent le financement sont eux-mêmes caractérisés par des évolutions fortement structurantes. Les exigences de ce que l'on désigne sous le terme générique d'édition électronique dépassent très largement celles qui sont requises pour une simple mise en ligne des contenus rédactionnels. Pour le dire autrement, il s'agit désormais de développer une édition scientifique authentiquement numérique et pas seulement une production éditoriale à l'aide du numérique. $L a$ visibilité et, à terme, la viabilité éditoriale de tous les périodiques scientifiques ne pourront plus se concevoir sans sinscrire dans ce qui est authentiquement un nouveau paradigme, à la fois éditorial, scientifique et économique. Le corollaire en est que la publication de revues scientifiques requiert aujourd'hui des compétences techniques et humaines éprouvées, nouvelles et spécifiques. La société Acalis, dont ce n'est pas le coeur du métier, n'était pas en mesure de mettre à la disposition de notre journal de telles nouvelles ressources dans un délai prévisible. C'est donc en plein accord avec leurs responsables - et avec eux - que les mandataires du comité de rédaction et du conseil d'administration de la Société internationale francophone d'éducation médicale (SIFEM), dont le journal est l'organe officiel d'expression, ont recherché de nouveaux partenaires, en mesure de répondre à ces nouveaux défis.

Ensemble, nous avons choisi de déléguer désormais l'édition de la revue à la maison d'édition EDP Sciences (Édition Diffusion Presse Sciences), très liée au monde scientifique, filiale de la Société française de physique et partenaire de plusieurs autres sociétés savantes dans les domaines de l'astrophysique, de la physique fondamentale et appliquée, des mathématiques, de l'électronique, des sciences des matériaux, des sciences de la vie, ainsi que dans le domaine de la santé. Le choix de cet éditeur a notamment été guidé par sa grande expertise dans le champ de l'édition électronique et de la gestion des bases de données. Il publie actuellement près d'une quarantaine de revues en ligne et exploite aussi un portefeuille de plus de 400 livres. Grâce aux ressources qu'il propose, de 


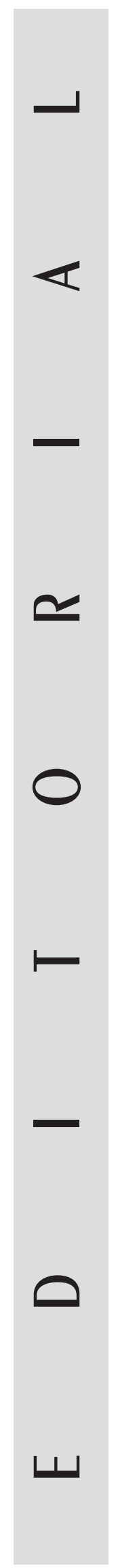

nouvelles fonctionnalités seront désormais offertes aux abonnés, via un site Internet redéfini. Dans le prochain numéro de la revue, une rubrique présentera plus complètement à ceux qui n'en sont pas encore familiers ce qu'est l'identifiant DOI ${ }^{\circledR}$ (Digital Objet Identifier), qui permet aux articles d'être identifiables par les moteurs de recherche des bases de données internationales mais aussi d'être mis en ligne et citables dès validation, avant même leur compilation dans un numéro complet, ou encore le système CrossRef, qui permet au lecteur de cliquer sur la majorité des références annexées à chaque article et d'avoir ainsi accès à l'article cité, pour autant que lui-même ou sa bibliothèque institutionnelle soient abonnés à la revue concernée.

Ces différentes fonctionnalités sont désormais indispensables et constitueront un nouvel atout majeur sur le chemin de l'indexation dans les grandes bases de données bibliographiques internationales (MEDLINE, EMBASE, ISI Journal Citation Reports, etc.), condition nécessaire pour la valorisation scientifique et la reconnaissance des travaux produits dans le champ de l'éducation des sciences de la santé. Un dispositif de soumission en ligne des articles déchargera les membres du comité de rédaction des fastidieuses et répétitives tâches de secrétariat, facilitera les relations avec les lecteurs-experts et avec les auteurs, qui pourront sinformer en ligne du suivi du processus d'évaluation éditoriale du manuscrit, et raccourcira les délais de décision éditoriale. Une disponibilité accrue pour les tâches essentielles de soutien scientifique et d'assistance éditoriale auprès des auteurs sera ainsi permise, au service de l'une des missions essentielles de la SIFEM et de sa revue.

La revue papier persistera simultanément; dès le numéro d'automne, elle paraîtra avec une maquette intérieure réactualisée pour prendre en compte ces nouvelles fonctionnalités. A partir du tome 10, en février 2009, elle sera distribuée sous une couverture rénovée, plus informative, qui préservera cependant l'esprit et les couleurs de la charte graphique initiale, car il ne saurait être question de priver "la revue verte " de la fonction identificatrice d'une livrée élégante, qui saffirme au fil des parutions. En contrepartie de ces nouveaux services, les tarifs des différents abonnements - aux versions papier etlou

numérique - et des abonnements couplés aux adhésions à la SIFEM seront réajustés - avec tact et mesure -, pour consolider une viabilité économique encore fragile et pour donner à la société les justes moyens au service de ses nouvelles ambitions: rapprochement avec les structures homologues francophones (Conférence internationale des doyens et des facultés de médecine d'expression française - CIDMEF - et ses Journées universitaires francophones de pédagogie des sciences de la santé, Université médicale virtuelle francophone-UMVFet son colloque Internet et pédagogie des sciences de la santé et du sport-IP3S -, Forum international francophone de pédagogie des sciences de la santé), européennes (Association for Medical Education in Europe) ou nord-américaines (Association canadienne pour l'éducation médicale - ACEM -), mais aussi production de documents didactiques et de recommandations, développement de structures et de réseaux de formation pédagogique ou de formation à la recherche en éducation des sciences de la santé, etc.

Ainsi, au-delà des relations humaines qui ont été nouées et qui demeureront, des chemins vont se séparer et d'autres converger. Enrichis et confortés par le soutien de nos partenaires éditoriaux historiques, auxquels nous exprimons à nouveau notre plus sincère reconnaissance, c'est avec confiance que nous nouons de nouvelles alliances et que nous inscrivons les projets de la communauté francophone en éducation des sciences de la santé dans l'avenir.

Jean JOUQUAN

Rédacteur en chef Mailto:jean.jouquan@chu-brest.fr

Jean-Luc DEBRU Président de la SIFEM Mailto:JLDebru@chu-grenoble.fr 